controls after a full explanation of the study. Clinical assessment of disease activity by SLE Disease activity index (SLEDAI) [3]. Lupus nephritis was assessed clinically with the renal SLE disease activity index (rSLEDAI).Indicated renal biopsies were taken from the patients with LN and were classified according to the International Society of Nephrology and the Renal Pathology Society (ISN/RPS) classification [4].Serum and urinary levels of TWEAK were measured for the patients and controls using enzyme-linked immunosorbent assay (ELISA). Fn 14 was examined in renal biopsies from LN group by immunohistochemistry.

Results: A significantly higher UTWEAK level on comparing SLE patients with LN to those without $L N$ and controls ( $F=149.2, P<0.001)$. UTWEAK had a highly significant positive correlation with, proteinuria $(r=0.755, P<0.001)$, a significant positive correlation with SLEDAI and rSLEADI $(r=0.217, P<0.037)(r$ $=0.476, P<0.024)$ respectively. UTWEAK had a significant negative correlation with anti-dsDNA titres, C3 and C4 $(r=-0.579, P<0.008)(r=-0.456, P<0.011)$ $(r=-0.552, P<0.002)$. Although sTWEAK level was higher in SLE patients than controls, it was not found to be associated with the presence of $L N(F=4.963$, $\mathrm{P}=0.012)$. Fn14 expression was detected in glomerular and tubular cells in LN patients.


Expression of Fn14 in renal biopsies from LN patients was examined. Immunohistochemistry for Fn14 was detected in (A) glomerular endothelial cells, $(B, C)$ some specimens showed moderate and intense staining for Fn14 in renal tubular cells. In the controls [renal biopsies from patients with renal cell carcinoma (D)], there was very slight staining for Fn14 in renal tubular cells.

Conclusion: Urinary TWEAK is a specific and sensitive biomarker for detection of active LN in newly diagnosed untreated SLE patients.

References:

[1] Kwok SK and Tsokos GC. New insights into the role of renal resident cells in the pathogenesis of lupus nephritis. Korean J Intern Med 2018; 33 (2): 284-289.

[2] Xia Y, Herlitz LC, Gindea S, Wen J, Pawar RD, Misharin A, et al. Deficiency of Fibroblast Growth Factor-Inducible 14 (Fn14) Preserves the Filtration Barrier and Ameliorates Lupus Nephritis. J Am Soc Nephrol 2015; 26(5):1053-1070.

[3] Bombardier C, Gladman DD, Urowitz MB, Caron D, Chang CH, Derivation of the SLEDAI. A disease activity index of lupus patients. Arthritis Rheum 1992; 35: $630-40$.

[4] Weening JJ, D'Agati VD, Schwartz MM, Seshan SV, Alpers CE, Appel GB, et al. The classification of glomerulonephritis in systemic lupus erythematosus revisited. J Am Soc Nephrol 2004; 15(2): 241-250.

Disclosure of Interests: None declared

DOI: 10.1136/annrheumdis-2020-eular.2196

\section{AB0399 \\ SYSTEMIC LUPUS ERYTHEMATOSUS AND OPPORTUNISTIC INFECTIONS: PREVALENCE, FEATURES OF CLINICAL SYMPTOMS}

B. Belov ${ }^{1}$, O. Egorova ${ }^{1}$, D. Movsisyan ${ }^{2} .{ }^{1}$ V.A. Nasonova Research Institute of Rheumatology, Moscow, Russian Federation; ${ }^{2}$ Pirogov Russian National Research Medical University, Moscow, Russian Federation
Background: Modern therapy for systemic lupus erythematosus (SLE) is associated with long-term treatment with cytotoxic drugs which is often accompanied by activation of comorbid infection, including viral infection.

Objectives: to identify prevalence and specific characteristics of the clinica symptoms of co-infection in SLE.

Methods: 67 patients with SLE were examined, mainly middle-aged women (62) of $33.5 \pm 8.1$ years old with the disease duration of 1 to 7 years who received "basic therapy". All patients were examined by ELISA for the presence of Herpesviridae viruses.

Results: In 67 patients, ELISA revealed anti-viral antibodies IgG to CMV $(86.5 \%)$ and VCA IgG of EBV $(56.7 \%)$, somewhat less frequently than HSV-1 IgG (38.8\%) and HSV-2 (26. $8 \%$ ). Active CMV and EBV infections were diagnosed in $26(38.8 \%)$ and $19(28.3 \%)$ patients, respectively; moreover, in 11 patients a combination of production of antibodies of the IgM CMV and EA IgG EBV class was revealed. Analysis of anamnestic data and serological examination allowed us to distinguish 3 groups of patients: group I- 35 patients with a viral infection, including 9 with the infection of a mixed viral and bacterial nature; Group II - 14 patients with bacterial infection and Group III - 18 patients without viral and bacterial complications. Analysis of clinical symptoms showed that there is a definite correlation of high titers of antibodies to CMV and EBV with symptoms such as fever $(p<0.01, r=0.74)$, polyarthralgia $(p<0.02, r=0.46)$ lymphadenopathy $(p<0.01, r=0.74)$, carditis $(p<0.05, r=0.42)$, hepatomegaly $(p<0.05, r=0.62)$, central nervous system damage $(p<0.02, r=0.46)$, migratory erythematous/hemorrhagic rash $(p<0.05, r=0.58)$, urinary syndrome $(p<0.02, r=0.41)$, anti-dsDNA $(p<0.01$, $r=0.82)$, ANA $(p<0.02, r=0.74)$; cryoprecipitins $(p<0.01, r=0.45)$. Although there were similar clinical manifestations, the presence of CMV and EBV had some organ specificity. Thus, damage to the central nervous system joints (polyarthralgia) and liver was more common in patients with CMV, and lymphadenopathy and erythematous/hemorrhagic rash - in patients with EBV.

Conclusion: In SLE a comorbid viral infection contributes to clinical picture with a lingering remitting inflammatory process, as well as insufficient effectiveness from corticosteroid and immunosuppressive therapy.

Disclosure of Interests: None declared

DOI: 10.1136/annrheumdis-2020-eular.1444

\section{AB0400 1 PATIENT-REPORTED OUTCOME MEASURES IN SWEDISH PATIENTS WITH RECENT-ONSET SLE VERSUS RA IN THE FIRST 60 MONTHS AFTER DIAGNOSIS}

M. Bjork ${ }^{1}$, I. Thyberg ${ }^{1}$, A. Kastbom ${ }^{1}$, R. Heijke ${ }^{1}$, L. Mcdonald ${ }^{2}$, E. Alemao ${ }^{3}$ C. Sjowall ${ }^{1}{ }^{1}$ Linköping University, Linköping, Sweden; ${ }^{2}$ Bristol-Myers Squibb, Uxbridge, United Kingdom; ${ }^{3}$ Bristol-Myers Squibb, Princeton, United States of America

Background: Patient (pt)-reported outcome measures (PROMs) provide information on a pt's own perception of disease impact, helping to provide a global perspective of disease when combined with conventional physician assessments. There is a discrepancy in PROMs between pts with rheumatoid arthritis (RA) and those with systemic lupus erythematosus (SLE); improvements in PROMs are often seen within the first year after diagnosis among pts with RA, but not SLE. ${ }^{2}$ Whether this discrepancy persists during subsequent years is unknown

Objectives: To compare changes in PROMs among pts with SLE versus RA within the first 60 months after diagnosis.

Methods: Pts with SLE with no prior organ damage were consecutively enrolled (2010-2015) from the Clinical Lupus Register in Northeastern Gothia and met the $\geq 4$ of the 1982 American College of Rheumatology (ACR) and/or the 2012 Systemic Lupus International Collaborating Clinics classification criteria. Pts with RA were included from the observational 2nd Management of Early Intervention in RA cohort (TIRA-2; 2006-2009), ${ }^{3}$ which enrolled pts with recent-onset RA; $84 \%$ of patients fulfilled the 1987 ACR criteria. Pts in both cohorts had symptoms for $<12$ months prior to diagnosis/inclusion and were treated according to Swedish guidelines. Pts with SLE or RA were followed prospectively after diagnosis. PROMs (quality of life: EuroQoL-5 Dimensions [EQ-5D]; activity limitations: Health Assessment Questionnaire [HAQ]; pain, fatigue, and well-being: Visual Analogue Scale $0-100 \mathrm{~mm}$ ) were collected at Months 0 (inclusion), 6, 12, 24, 36, 48, and 60. HAQ, pain, fatigue, well-being: higher scores indicate greater severity; EQ-5D: lower scores indicate greater severity. 
Table. Patient characteristics at registry entry.

\begin{tabular}{lll}
\hline Characteristic & $\begin{array}{l}\text { SLE } \\
(\mathbf{n = 4 1 )}\end{array}$ & $\begin{array}{l}\mathbf{R A} \\
\mathbf{( n = 5 2 2 )}\end{array}$ \\
\hline Age, median (interquartile range), years & $39.0(18-77)$ & $60.0(51-68)$ \\
Female sex & $33(80.5)$ & $352(67.4)$ \\
White & $35(85.4)$ & $\mathrm{NC}$ \\
Previous or current tobacco smoking & $18(43.9)$ & $183(65.6)^{\star}$ \\
Fulfilled 1982 ACR SLE classification criteria & $36(87.8)$ & $\mathrm{NA}$ \\
Number of fulfilled ACR SLE criteria, mean (range) & $4.7(3-9)$ & $\mathrm{NA}$ \\
Fulfilled 2012 SLICC classification criteria & $41(100)$ & $\mathrm{NA}$ \\
Fulfilled 1987 ACR RA criteria & $\mathrm{NA}$ & $439(84.1)$ \\
\hline
\end{tabular}

Data are $\mathrm{n}(\%)$ unless otherwise specified.

${ }^{*} \mathrm{n}=279$.

$\mathrm{ACR}=$ American College of Rheumatology; $\mathrm{NA}=$ not available; $\mathrm{NC}=$ not collected due to ethics approval; RA=rheumatoid arthritis; SLE=systemic lupus erythematosus; SLICC=Systemic Lupus International Collaborating Clinics.

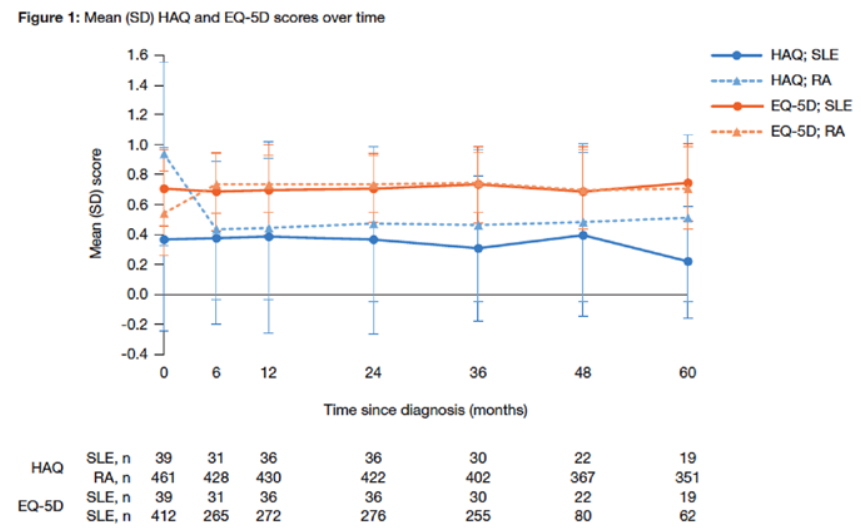

HAQ: higher scores indicate greater severity. EQ-5D: lower scores indicate greater severity. Patients with missing data were not included in the mean (SD) calculations for that particular time point. RA-5D=EuroQoL-5 Dimensions; $\mathrm{HAQ}=$ Health Assessment $\mathrm{Q}$

Results: 41 pts with SLE and 522 pts with RA were included in this analysis. Numerical differences between cohorts in age, sex, and tobacco smoking were seen (Table). Baseline PROM scores were generally worse for pts with RA versus SLE (Figs 1 and 2). However, an improvement in PROM scores was seen by Month 6 for pts with RA, but not SLE. Between Months 6 and 60, PROM scores remained largely unchanged for both groups.

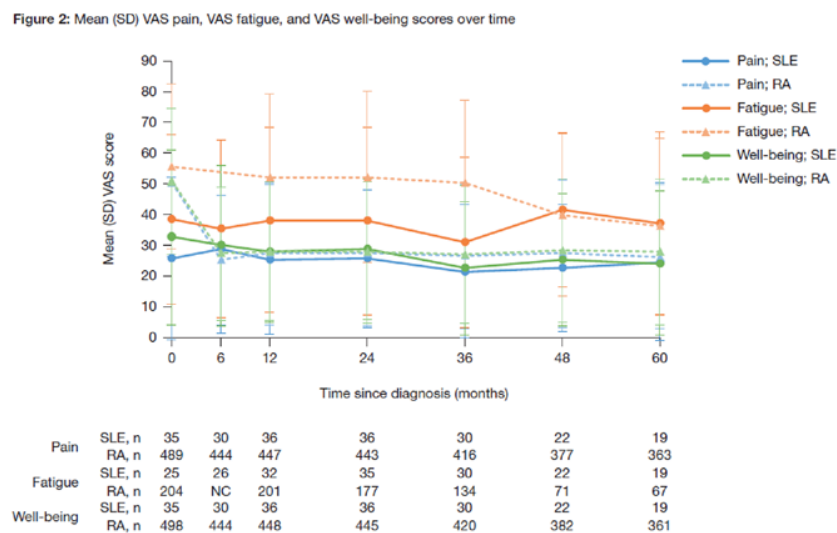

Pain, fatigue, and well-being (NAS ranging from 0-100): higher scores indicate greater severity. VAS fatigue data were not collected for patients with
$\mathrm{NC}=$ not collected due to ethics approval; AS =Visual Analogue Scale.

Conclusion: PROMs in pts with early SLE tended to remain stable in the years following diagnosis compared with the improvement experienced by pts with early RA, indicating a greater unmet need in SLE. The lack of improvement in PROMs in pts with SLE may be due to the disease's impact across multiple organ systems, which may take longer to resolve versus RA symptoms. These results imply there is room for improvement in disease management - both pharmacological and multi-professional interventions. Data should be interpreted with caution due a low number of pts with SLE.

References:

[1] Versteeg GA et al. Clin Rheumatol. 2018;37:1189-1197.

[2] Heijke R et al. Arthritis Rheumatol. 2018;70 (suppl 10):abstract 2633.

[3] Hallert E et al. Scand J Rheumatol. 2015;44:100-105.

Acknowledgments: Sreeram Ramagopalan (supervised analysis). Lola Parfitt (medical writing, Caudex; funding: BMS)

Disclosure of Interests: Mathilda Bjork: None declared, Ingrid Thyberg: None declared, Alf Kastbom Consultant of: Bristol-Myers Squibb, Pfizer, Roche, UCB Employee of: Sanofi, Speakers bureau: Bristol-Myers Squibb, UCB, Rebecca Heijke: None declared, Laura McDonald Shareholder of: Bristol-Myers Squibb, Employee of: Bristol-Myers Squibb, Evo Alemao Shareholder of: Bristol-Myers Squibb, Employee of: Bristol-Myers Squibb, Christopher Sjowall: None declared DOI: 10.1136/annrheumdis-2020-eular.321 